\title{
一类非保守摆方程拟周期解的存在性 和所有解的有界性
}

\author{
尤建 功 \\ (南京大学数学系,南京 210008)
}

关链词抧期解、有界性、可逆系统

本文考虑下列形式的二阶微分方程

$$
\frac{\mathrm{d}^{2} x}{\mathrm{~d} t^{2}}+F_{x}^{\prime}(t, x) \frac{\mathrm{d} x}{\mathrm{~d} t}+G_{x}^{\prime}(t, x)=0,
$$

这里 $F(t, x), G(t, x) \in C^{\infty}\left(\mathbf{R}^{2} / \mathbf{Z}^{2}\right)$. 方程 (1) 等价于系统

$$
\frac{\mathrm{d} x}{\mathrm{~d} t}-y-F(t, x), \frac{\mathrm{d} y}{\mathrm{~d} t}-G_{x}^{\prime}(t, x) .
$$

设 $F(t, x), G(t, x)$ 满足下面的对称性条件:

$$
F(-t,-x)-F(t, x), G(-t,-x)-G(t, x) .
$$

由于 $F(t, x)$ 和 $G(t, x)$ 均为 $x$ 的周期函数, 系统 (2) 可以看作柱面上的非自治系统. 当 $F(t, x)=0$ 时, 方程 (2) 为保守系统. 当 $F(t, x) \neq \equiv$ 时, (2) 式不再是保守系统. 这 不同于文献 [1], 从而 Moser 的扭转定理不再适用.

定理 1 如果系统 (2) 满足对称性条件 (3), 则 (2) 式在 $(t, x, y)$ 空间 $\mathrm{R}^{2} / \mathrm{Z}^{2}$ 中存 在无穷多个不变环面, 且不变环面上的解均为拟周期解. 进一步有系统 (2) 在相柱面上没有 无界解, 即对 (2) 式的任一解 $(x(t), y(t))$, 存在常数 $M$, 使得 $\sup _{t \in \mathbb{R}^{\mathbf{R}}}|y(t)|<M$.

令 1 为 $\mathbf{R}^{1}$ 中开区间, 记 $\bar{A}-\mathbf{R}^{1} \times I, \bar{A}$ 到 $\mathbf{R}^{2}$ 的映射 $\bar{P}$ 为

$$
\left\{\begin{array}{l}
x_{1}-x+y+f_{1}(x, y), \\
y_{1}-y+f_{2}(x, y),
\end{array}\right.
$$

这里 $f_{1}(x, y), f_{2}(x, y)$ 均为 $x$ 的 1-周期函数, 且 $f_{1}(x, y), f_{2}(x, y) \in C^{\infty}\left(\mathbf{R}^{1} \times I\right)$. 记 $\bar{G}$ : $\bar{A} \rightarrow \bar{A}$ 为对合映射 $(x, y) \mapsto(-x, y)$. 如果 $\bar{G} \circ \bar{P} \circ \bar{G}-\bar{P}^{-1}$, 则称 $\vec{P}$ 为可逆映射.

引理 $1^{[2,3]}$ 设 $\bar{P}$ 为 $\bar{A}$ 到 $\mathrm{R}^{2}$ 的形如 (4) 式的 $C^{\infty}$ 可逆映射, 则存在 $8>0$, 使得当

$$
\left|f_{1}\right|_{c^{-}}+\left|f_{2}\right|_{c^{-}}<\delta
$$

时,对每一个满足 Diophantine 条件

$$
|n \lambda-m| \geqslant \beta|n|^{-\gamma}
$$

的无理数 $\lambda \in I$ (这里 $n, m$ 为整数, $n \neq 0, \beta>0, r>1$ ), 都存在一个闭曲线 $\Gamma$, 使得 $\Gamma$ 在 $\vec{P}$ 下不变, 且 $\vec{P}$ 限制在 $\Gamma$ 上共轭于旋转数为 $\lambda$ 的旋转: $x \mapsto x+\lambda$ (这里 $T$ 为 $\Gamma$ 的提升)。

令 $\lambda_{0}>0$. 记

本文1990年4月2 日收到. 1991 年. 1 月 30 日收到修改稆. 


$$
A_{\lambda_{0}}:=\left\{(t, \theta, \lambda) \mid(t, \theta) \in \mathbf{R}^{2} / \mathbf{Z}^{2}, \lambda \geqslant \lambda_{0}\right\} .
$$

下面的引理中均假设 $F(t, x), G(t, x) \in C^{\infty}\left(\mathbf{R}^{2} / \mathbf{Z}^{2}\right)$ 且满足条件 (3).

引理 2 对充分大的 $\nu$, 存在变换 $\Psi$ :

$$
\left\{\begin{array}{l}
x=u+U(t, u, v), \\
y=v+V(t, u, v),
\end{array}\right.
$$

使得 $U(t, u, v), V(t, u, v) \in C^{\infty}\left(\mathbf{R}^{2} / \mathbf{Z}^{2} \times \mathbf{R}^{+}\right)$满足

$$
A_{0}+\subset \Psi\left(A_{v_{0}}\right) \subset A_{0}-\text {, }
$$

这里 $v^{+}>v_{0}>v^{-} \gg 1$. 并且对充分大的 $\nu, \Psi$ 将 (2) 式变为系统

$$
\frac{\mathrm{d} u}{\mathrm{~d} t}-v+h_{1}(t, u, v), \frac{\mathrm{d} v}{\mathrm{~d} t}-h_{2}(t, u, v),
$$

这里 $h_{1}(-t,-u, v)-h_{1}(t, u, v), h_{2}(-t,-u, v)--h_{2}(t, u, v)$.

证 对充分大的 $v$, 构造变换

$$
\left\{\begin{array}{l}
u=x+\frac{1}{v} \int_{0}^{x} F(t, s) \mathrm{d} s, \\
y=v-\frac{1}{v} G(t, x)
\end{array}\right.
$$

不失一般性, 可设 $F(t, x)$ 的均值为零, 从而 $\int_{0}^{*} F(z, s) \mathrm{d} s \in C^{\infty}\left(\mathrm{R}^{2} / \mathrm{Z}^{2}\right)$. 对充分大的 $\nu$, 我们 可由(8)式的第一个方程解得 $x-u+U(t, u, v)$, 其中 $U(t, u, v) \in C^{\infty}\left(\mathbf{R}^{2} / \mathbf{Z}^{2} \times \mathbf{R}^{+}\right)$. 将 其代人到 (8) 式的第二个方程就得到我们所求的变换 $\Psi$ :

$$
\left\{\begin{array}{l}
x-u+U(t, u, v), \\
y=v+V(t, u, v):=v-\frac{1}{v} G(t, u+U) .
\end{array}\right.
$$

$\Psi$ 将系统 (2) 变为

$$
\frac{\mathrm{d} u}{\mathrm{~d} t}=v+h_{1}(t, u, v), \frac{\mathrm{d} v}{\mathrm{~d} t}=h_{2}(t, u, v),
$$

这里

$$
\begin{aligned}
h_{2}(t, u, v)= & \frac{1}{v}\left(1+\frac{1}{v} G(t, x)\right)^{-1}\left(G_{t}^{\prime}(t, x)\right. \\
& \left.-G_{x}^{\prime}(t, x) F(t, x)-\frac{1}{v} G_{x}^{\prime}(t, x) G(t, x)\right), \\
h_{1}(t, u, v)= & \frac{1}{v}\left[\int_{0}^{x} F_{i}^{\prime}(t, s) \mathrm{d} s-F^{2}(t, x)-G(t, x)\right. \\
& \left.-\frac{1}{v} F(t, x) G(t, x)-\frac{1}{v} \int_{0}^{x} F(t, s) \mathrm{d} s \cdot h_{2}(t, s, v)\right],
\end{aligned}
$$

这里 $x-u+U(t, u, v)$.

由 (8) 式知,当条件 (3) 满足时,

$$
U(-t,-u, v)--U(t, u, v), V(-t,-u, v)-V(t, u, v),
$$

从而由 (10)、(11) 式有

$$
\begin{aligned}
& h_{1}(-t,-u, v)-h_{1}(t, u, v), \\
& h_{2}(-t,-u, v)--h_{2}(t, u, v) .
\end{aligned}
$$


引理 3 对函数 $h_{1}(t, u, v)$ 和 $h_{2}(t, u, v)$, 我们有下面的估计式:

$$
\left|h_{i}(t, u, v)\right|_{c^{-}}-O\left(\frac{1}{v}\right), i-1,2 \text {, }
$$

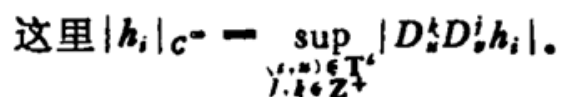

证由 (10) 式易见, $\left|h_{i}(t, u, v)\right|-O\left(\frac{1}{v}\right)$. 为了估计 $h_{i}(t, u, v)$ 的导数, 我们先 证明

$$
\begin{gathered}
\mid U\left(t, u,\left.v\right|_{c^{-}}+\mid V\left(t, u, v \mid c^{-}-O\left(\frac{1}{v}\right) .\right.\right. \\
\text { 令 } g_{1}(t, x, v)-\frac{1}{v} \int_{0}^{*} F(t, s) d s, g_{2}(t, x, v)--\frac{1}{v} G(t, x) \text {, 则 } \\
u-x+g_{1}(t, x, v) .
\end{gathered}
$$

将 (9) 式的第一个方程代人上式得

$$
U-g_{1}(t, u+U, v),
$$

将算子 $D \xi D^{i}$ 作用到 (14) 式两边. 用归纳法可以证得 $\left|D^{k} D_{\nu}^{i} U\right|-O\left(\frac{1}{v}\right), \forall i, k \in \mathrm{Z}^{+}$成 立, 从而 $|U(t, u, v)|_{c^{-}}-O\left(\frac{1}{v}\right)$ (类似于文献[1]性质 2.1 的证明). 注意到

$$
V(t, u, v)--\frac{1}{v} G(t, u+U) \text {. }
$$

直接求导可得估计式:

$$
|V(t, u, v)|_{c^{-}}-O\left(\frac{1}{v}\right) \text {. }
$$

将 $x-u+U(t, u, v)$ 代入 (10) 式,直接计算同样可得

$$
\left|h_{i}(t, u, v)\right|_{c^{-}}-O\left(\frac{1}{v}\right) \text {. }
$$

证毕.

记 $\phi^{t}(u, v)-(\bar{u}(t, u, v), \bar{v}(t, u, v))$ 为 (7) 式的初值 $\phi^{0}(u, v)-(u, v)$ 的解, 这 里 $v$ 充分大. 易知 $\phi^{1}:(u, v) \mapsto \phi^{1}(u, v)$ 为系统 (7) 的 Poincare 映射. 由引理 2 知, $G \circ \phi^{i} \circ G-\phi^{-t}$, 从而 $G^{\circ} \phi^{1} \circ G-\phi^{-1}-\left(\phi^{1}\right)^{-1}$, 即 $\phi^{1}$ 为可逆映射. 并且 $\phi^{1}$ 有下列形式:

$$
\begin{aligned}
& u_{1}-u+v+\int_{0}^{1} h_{1}(t, \bar{u}, \bar{v}) \mathrm{d} t+\int_{0}^{1} \mathrm{~d} t \int_{0}^{t} h,(s, \bar{u}, \bar{v}) \mathrm{d} s, \\
& v_{1}-\nu+\int_{0}^{1} h_{2}(t, \bar{u}, \bar{v}) \mathrm{d} t,
\end{aligned}
$$

这里 $(\bar{u}, \bar{v})-(\bar{u}(t, u, v), \bar{v}(t, u, v))$ 是 (7) 式的初值为 $(u, v)$ 的解. 令

$$
\begin{aligned}
& F_{1}(u, v)-\int_{0}^{1} h_{1}(t, \bar{u}, \bar{v}) \mathrm{d} t+\int_{0}^{1} \mathrm{~d} t \int_{0}^{t} h_{2}(s, u \bar{u}, \bar{v}) \mathrm{d} s, \\
& F_{2}(u, v)-\int_{0}^{1} h_{2}(t, \bar{u}, \bar{v}) \mathrm{d} t,
\end{aligned}
$$

则 (7) 式的 Poincare 映射 $\phi^{1}$ 可写为下列形式:

$$
u_{1}-u+v+F_{1}(u, v), v_{1}=v+F_{2}(u, v) \text {. }
$$

引理 4 对 $i-1,2$, 我们有

$$
\left|D_{i}^{i} D_{k}^{t} F_{i}(u, v)\right|-O\left(\frac{1}{v}\right), i, k-0,1,2 \cdots \text {. }
$$




\section{证类似于文献[1]中的引理 3.1. 略.}

定理 1 的证明 由引理 2-4, 当 $v$ 充分大时, 系统 (7) 的 Poincare 映射 $\phi^{1}$ 满茫引理 1 的所有条件. 从而当 $\lambda$ 充分大且满足无理性条件 (5) 时,存在 $\phi^{1}$ 的不变圆

$$
\Gamma: s \in S^{1} \rightarrow\left(\gamma_{1}(s), \gamma_{2}(s)\right) \text {. }
$$

且 $\phi^{3}$ 限制在 $r$ 上共轭于旋转数为 $\lambda$ 的旋转, 即 $\phi^{1} \circ \Gamma(s)-\Gamma(s+\lambda)$. 系统 (7) 在时刻 $t=0$ 时从 $\Gamma$ 上出发的解确定了一个不变环面

$$
T_{2}^{2}-\left\{\left(t, \vec{u}\left(t, \gamma_{1}(s), \gamma_{2}(s)\right), v\left(t, \gamma_{1}(s), \gamma_{2}(s)\right) \mid(t, s) \in \mathbf{R}^{2} / \mathbf{Z}^{2}\right\} .\right.
$$

记 $\tilde{\Psi}$ 为下面的变换:

$$
t=t, x=u+U(t, u, v), y=v+V(t, u, v),
$$

则 $\tilde{\Psi} \circ T_{2}^{2} \circ \tilde{\Psi}^{-1}$ 是系统 (2) 在空间 $(t, x, y) \in \mathbf{R}^{2} / \mathbf{Z}^{2} \times \mathbf{R}^{1}$ 中的不变环面. 且 $\tilde{\Psi} \circ T_{1}^{2} \circ \tilde{\Psi}^{-1}$ 上 的每一个解都是频率为 $(1, \lambda)$ 的拟周期解.

同理可得 (2) 式存在频率为 $(1,-\lambda)$ 的不变环面, 从而对任意的 $\left(x_{0}, y_{0}\right) \in S^{1} \times \mathbf{R}^{1}$, 系 统 (2) 有两个频率 $|\lambda|>\left|y_{0}\right|$ 的不变环面 $T_{ \pm 2}^{2}$. 因而对系统 (2) 初值为 $\left(x_{0}, y_{0}\right)$ 的解 $(x(t$, $\left.\left.x_{0}, y_{0}\right), y\left(t, x_{0}, y_{0}\right)\right)$, 有

$$
\sup _{t \in \mathbb{R}}\left|y\left(t, x_{0}, y_{0}\right)\right|<\lambda+K_{0}
$$

这里

$$
K_{0}-\sup _{(1, x) \in T^{4}}\left|G_{x}^{\prime}(t, x)\right|+1,
$$

因此系统 (2) 的所有解均为有界皱.

\section{今 考神}

[1] You Jiangong, J Differential Equations, 85(1990), 1:54-65

\{2] Moser, J., Ann. it Malh. Studies, No.77, Princeton. N. J., 1973.

131 Sevryuk, M., Lecture Notes in Mathematics, 1986, 1211. 